Política y Sociedad

ISSN: $\quad 1130-8001$

ISSN-e: $1988-3129$

\title{
Repertorios interpretativos de un manual de intervención en la infancia temprana en Chile
}

\author{
Claudia Calquín Donoso, ${ }^{1}$ Rodrigo Guerra Arrau, ${ }^{2}$ Katherine Escobar Villalobos ${ }^{3}$ y Javier \\ Martínez Riquelme ${ }^{4}$
}

Recibido: 02-06-2018 / Aceptado: 21-01-2020

Resumen. Si bien los estudios de gubernamentalidad se han erigido como una alternativa teórica y metodológica relevante para estudiar las políticas sociales, son pocos los trabajos que se han dedicado a estudiar las políticas específicas en la infancia. Como una forma de aportar a la investigación sobre la relación entre gubernamentalidad e infancia, se presentan los resultados de una investigación dirigida a conocer los repertorios interpretativos de un manual de atención psicosocial en el sistema de protección de la infancia en Chile. La metodología consistió en un análisis discursivo del cuerpo del manual basado en la perspectiva de los repertorios interpretativos. Se identificaron cuatro repertorios: 1) cimientos de lo social: la relación como actor; 2) el Estado como agente de racionalidad y salvación nacional; 3) la operación del manual: el Estado como coach, y finalmente 4) la figura de la madre: del sujeto en riesgo al sujeto de riesgo. Se concluye que la producción discursiva de la infancia opera por medio de prácticas discursivas cuya función es su despolitización, bajo grillas interpretativas de corte naturalístico que opacan las relaciones de poder, económicas y de saber que cruzan la pobreza infantil y las tensiones entre el saber científico y el saber empírico de las madres.

Palabras clave: infancia; discurso; política gubernamental; gubernamentalidad; análisis de discurso.

\section{[en] Interpretative Repertoires about an Intervention Manual in Early Childhood}

\footnotetext{
1 Universidad Alberto Hurtado (Chile)

E-mail: ccalquindonoso@gmail.com

2 Universidad Alberto Hurtado (Chile)

E-mail: rodrigo.guerraarrau@gmail.com

3 Universidad Alberto Hurtado (Chile)

E-mail: escobar.katherine23@gmail.com

4 Universidad Alberto Hurtado (Chile)

E-mail: javier.martinez.uah@gmail.com
}

Abstract. Although governmentality studies have emerged as a relevant theoretical and methodological alternative for studying social policies, few studies have focused on the study of specific policies in childhood. As a way to contribute to research on the relationship between governmentality and childhood, we present the results of research aimed at understanding the interpretative repertoires of a psychosocial care manual for a child protection system in Chile. The methodology consisted of a discursive analysis of the body of the manual based on the perspective of interpretive repertoires. Four repertoires were identified: 1) foundations of all that is social: the relationship as an actor; 2) the state as an agent of national rationality and salvation; 3) the 
operation of the manual: the state as coach; and finally, 4) the figure of the mother: from the subject at risk to the subject of risk. It is concluded that the discursive production of childhood operates through discursive practices whose function is its depoliticization, under naturalistic interpretive grids that obscure the power, economic and knowledge relations that permeate childhood poverty and the tensions between scientific knowledge and the empirical knowledge of mothers.

Keywords: childhood; discourse; government policy; governmentality; discourse analysis.

Cómo citar: Calquín Donoso, C. et al. (2020): "Repertorios interpretativos de un manual de intervención en la infancia temprana en Chile”, Política y Sociedad, 57(1), pp. 197-215.

Sumario. 1. Introducción. 2. La gubernamentalidad y el gobierno de la infancia pobre. 3. Tecnologías literarias: el Manual para el apoyo y seguimiento del desarrollo psicosocial de los niños y niñas de 0 a 6 años y la construcción de objetos y factualidades. 4. Material y método. 5. Resultados. 6. Conclusión y discusiones. 7. Bibliografía.

Agradecimientos. Artículo realizado en el marco del proyecto de investigación $\mathrm{N}^{\circ}$ CC71-03-17 "Gobernando la infancia pobre: repertorios interpretativos sobre la infancia y la crianza en los discursos del sistema de protección social Chile Crece Contigo", financiado por el Fondo de Investigación para profesores colaboradores de la Facultad de Psicología, Universidad Alberto Hurtado.

\section{Introducción}

En los últimos años, en Chile, los estudios de gubernamentalidad se han erigido como una alternativa epistemológica y metodológica que ha abierto nuevas interrogantes desde donde pensar las políticas públicas y sociales, al aportar cierto desplazamiento desde una mirada puesta en su evaluación y efectividad hacia lo que Foucault llamó sus "condiciones de posibilidad” (Foucault, 2008). Entre los distintos estudios destacan: en políticas de salud, Castillo (2016) y Soto (2016); en juventud, Mardones (2015) y Rivera (2016, 2016a); en mujeres, Galaz y Guarderas (2017); en urbanismo, Crisosto y Salinas (2016), y en educación, Lepe-Carrión (2015), Apablaza (2015), González, Ligueño y Parra (2015).

Pese a su potencial heurístico para comprender las múltiples maneras en que "diversos saberes han sido utilizados en la conducción de la población a través de cálculos, procedimientos y tácticas" (Chávez, 2016: 23), son casi inexistentes los trabajos que analizan las prácticas de gobierno específicas de la infancia, en un contexto en que la infancia se vuelve un sujeto prioritario de la política social y de salud mental chilena. Así el "gobierno de la infancia" puede ser un camino privilegiado para comprender las estrategias biopolíticas actuales en términos de los ensamblajes entre el Estado, la organización social del cuidado y la construcción de la infancia en las poblaciones empobrecidas.

En este trabajo presentamos los resultados de una investigación dirigida a conocer los repertorios discursivos que se despliegan en el Manual para el apoyo y seguimiento del desarrollo psicosocial de los niños y niñas de 0 a 6 años. Este está dirigido a profesionales que intervienen con familias usuarias del programa de Apoyo Biopsicosocial ejecutado por el Ministerio de Salud, programa eje del 
sistema de protección de la infancia Chile Crece Contigo (CCC $)^{5}$. La hipótesis que desplegaremos en este escrito es que este manual, en tanto dispositivo discursivo que establece diversas acciones que los profesionales deben ejecutar, puede ser considerado una instancia de realización de modos de existencia de la infancia. Lejos de describir o representar categorías preformadas en torno a la noción de infancia e intervención psicosocial, entre otras, construye versiones de estas; es decir, siguiendo la lógica arqueológica de Michel Foucault, el manual construye sus propios objetos (Foucault, 2011; Laclau y Mouffe, 2004), y es esta capacidad de acción del manual lo que concentra su performatividad.

Por otro lado, y de modo complementario, el manual puede ser entendido bajo lo que Shapin y Shaeffer (2005) llaman una "tecnología literaria", esto es: una herramienta que permite la construcción de una comunidad de expertos a través de la cual se dan a conocer fenómenos que toman la apariencia de un hecho objetivo. Esta acción se realizaría, entre otros recursos retóricos, por medio de la invocación de un conocimiento experto, fáctico y sin autoría. Así el análisis del manual, desde una perspectiva discursiva, nos separa de una posición estrictamente lingüística para introducirnos en el espesor material de las prácticas institucionales (Laclau y Mouffe, 2004) que producen y reproducen lo que llamaremos "la infancia pobre".

\section{La gubernamentalidad y el gobierno de la infancia pobre}

Los estudios en gubernamentalidad en las últimas décadas han sido incorporados de forma creciente como clave interpretativa de las políticas públicas en los contextos neoliberales. Para esta perspectiva, las prácticas de gobierno se comprenden en términos del vínculo entre los diversos dispositivos estatales y privados en la constitución y comprensión de sí mismo, y de este vínculo en términos de la gestión de la propia libertad (Foucault, 2012; Rose, 2007). De acuerdo a Foucault, la gubernamentalidad corresponde al "conjunto constituido por las instituciones, los procedimientos, análisis y reflexiones, los cálculos y las tácticas que permiten ejercer esa forma bien específica, aunque muy compleja, de poder que tiene por blanco principal la población" (Foucault, 2012: 136). La gubernamentalidad describe una forma "del intento de alcanzar fines sociales y políticos actuando de forma calculada sobre las fuerzas, actividades y relaciones de los individuos que constituyen una población" (Rose, 1990, 4).

Siguiendo a Rose (2007) la infancia, como uno de los espacios más gobernados en las democracias neoliberales, es el lugar por excelencia de la modelación del comportamiento humano. Esta cuestión nos conduce además a los modos en que la acción institucional - dirigida a eliminar las inequidades existentes en la infancia chilena y a mejorar el acceso a los servicios de salud, vivienda y educación, entre

\footnotetext{
El Sistema de Protección Chile Crece Contigo es un sistema integrado de intervenciones sociales, programas, prestaciones y servicios coordinado por el Ministerio de Desarrollo Social, y que recibe el aporte de diversos organismos públicos, como los Ministerios de Salud y de Educación, la Junta Nacional de Auxilio Escolar y Becas, la Junta Nacional de Jardines Infantiles y la Fundación Integra. Reúne 18 programas dirigidos a mujeres gestantes y niños hasta los 4 años. La misión es acompañar, proteger y apoyar a los niños, niñas y sus familias en el desarrollo psicosocial como forma de superar los rezagos del desarrollo de las clases más pobres, y entregar herramientas para la superación de la pobreza.
} 
otros - se ha desplazado hacia la incorporación e institucionalización de formas de conocimiento vinculados a la psicología y saberes afines (psiquiatría y psicoanálisis), por medio de la promoción sistemática del discurso de un personal experto en cuestiones de crianza y desarrollo infantil (Ehrenreich y English, 2010; Llobet, 2014).

La introducción de lo "psi" en la producción de ciudadanos de derechos (Rose, 1990, 2007) supone analizar el rol del campo de lo psicológico dentro de la cartografía del poder y de cómo la subjetividad y la esfera personal e íntima -ámbito propio del quehacer psicológico en su versión mainstream - entran en los cálculos políticos a través de una combinación de lo que Foucault (2012) llama técnicas disciplinarias y biopolíticas vinculadas, por un lado, a la producción de cuerpos dóciles y adaptados, y, por otro, a la gestión del riesgo por medio de la integración de novedosos ámbitos de problematización a una red de saberes y tecnologías.

Cabe destacar que este saber experto se entronca con dos cuestiones. Por un lado, con la incorporación de la teoría del capital humano en su versión fuerte. Así vemos que junto a los fundamentos psicológicos, hay una fuerte referencia en los mismos documentos del sistema a los trabajos de teóricos de la escuela de economía de la Universidad de Chicago, como a publicaciones del Banco Mundial. En ellos la intervención en la infancia temprana se ofrece como una inversión que permitiría la obtención de retornos económicos mayores que con las intervenciones que se realizan en etapas posteriores ${ }^{6}$. Por otro, entronca con lógicas de subsidiaridad a las instituciones privadas dedicadas al bienestar en ámbitos claves como la educación, la salud y la protección social, al profundizar una gubernamentalidad de tipo tecnocrático que si bien no es nueva, ha sido clave en el proyecto de modernización del Estado e internacionalización de la economía.

Así estos tecnócratas, entendidos como sujetos con un considerable entrenamiento académico que los habilitaría en la toma de decisiones o de consejería en grandes y complejas organizaciones, tanto públicas como privadas, "no solo han sido los principales implementadores de políticas y programas, sino que, además, han a menudo proporcionado a los movimientos políticos de las herramientas necesarias para articular sus proyectos de sociedad" (Silva, 2006: 179). Para Astete y Vaccari (2017), el rol de los expertos y la tecnocracia posibilitan escasas experiencias de participación y gobernanza ciudadana, cuestión que se vuelve problemática en un contexto en que los objetivos de las políticas sociales apuntan a la habilitación de espacios de autonomía y agenciamiento de las comunidades empobrecidas.

En el caso del programa de Apoyo al Desarrollo psicosocial del sistema de protección de la infancia CCC, estas intervenciones "psi" y de desarrollo de capital humano se dirigen a la promoción de la estimulación temprana, las competencias parentales y la crianza activa, todo esto bajo la égida de lo que ha llamado las "intervenciones en apego". Estas intervenciones se focalizan principalmente en la estimulación de la sensibilidad parental, en el fortalecimiento de la capacidad de

\footnotetext{
Los trabajo citados son Carneiro, P. y J. Heckman (2003) "Human Capital Policy", Discussion Paper, 821 y Fernald L., P. Kariger, P. Engle y A. Raikes (2009): "Examining Early Child Development in Low-Income Countries: A Toolkit for the Assessment of Children in the First Five Years of Life", Washington D.C., The World Bank.
} 
mentalización de los cuidadores y en brindar apoyo social a los padres (Gómez, Muñoz y Santelices, 2008). Como se destaca en el mismo manual, son la calidad y la frecuencia de las interacciones familiares (CCC, 2012) y el "capital relacional" (CCC, 2006) los que permiten al niño alcanzar su máximo nivel de desarrollo. Así leemos que:

(...) la falta de estimulación y de cuidados de adultos significativos en edades tempranas tiene efectos devastadores e irreversibles en el desarrollo de las funciones cerebrales, ya que se altera su organización y disposición frente al aprendizaje. Por el contrario, las experiencias ricas y positivas durante la primera infancia pueden tener efectos positivos en el desarrollo cerebral, ayudando a los niños/as en la adquisición del lenguaje, en el desarrollo de destrezas de resolución de problemas, la formación de relaciones saludables con iguales y adultos (CCC, 2006: 12).

Es necesario destacar que la neurociencia es uno de los saberes que actúa como grilla y marco de interpretación de las acciones del programa, por medio de la cual se explica el desarrollo infantil y, desde dicho programa, se implementan sus prácticas de intervención. Para el programa, las neurociencias permiten una mayor comprensión de los procesos de desarrollo cerebral y del sistema nervioso y sensorial, pues ponen en evidencia:

(...) la importancia vital de la vida intrauterina y de los primeros años en la maduración biológica, que permite a los individuos llegar a ser personas integradas en una sociedad. Así mismo, se confirma que esa maduración biológica depende y se produce en interacción con el ambiente humano y físico en el que el niño o la niña crece (CCC, 2012: 7).

Siguiendo a Rose (2007; Rose y Abi-Rached, 2013), la neurociencia es un dispositivo a través del cual es posible dirigir y modelar la infancia y el crecimiento infantil, en una combinación de bioingeniería cerebral y técnicas conductuales. Estas intervenciones no buscarían tanto normalizar un comportamiento desviado como "ajustar al individuo y restaurar y mantener su capacidad para participar en los circuitos de la vida cotidiana" (Rose, 2007: 89). En este sentido, podemos hablar de una neuropsicologización de la infancia que, según creemos, estructura y hace eficaz la matriz simbólica desde la cual las políticas neoliberales privatizan, despolitizan e individualizan los riesgos en la población infantil. Siguiendo a Bianchi (2012), el manejo del riesgo se desplaza de ser una empresa colectiva a una estrategia individual y familiar, recodificando la definición y el tratamiento de los problemas llamados psicosociales. Así, y de acuerdo a esta misma autora, "los individuos deben hacerse crecientemente responsables por el management de su propio destino y el de su familia, a través del cálculo de las consecuencias futuras de los actos presentes" (Bianchi, 2012: 87).

En este contexto de redefinición del riesgo social, el discurso experto y legitimado de los saberes "psi" — mediante la articulación de lenguajes, vocabularios, paradigmas argumentativos y modos de subjetivación - cumple la función de ser "un puente administrativo desde el Estado mediante el cual se despolitiza nuevamente un problema" (Llobet, 2014: 215), y cimenta cierta idea de 
que "el discurso de derechos es despolitizado por su interpretación psi" (Llobet, 2014: 225), y de manera similar, que los derechos del niño configuran un punto de toque para la individualización y psicologización del gobierno de la infancia (Llobet, 2014).

Vemos, por lo tanto, que las disciplinas "psi" juegan un papel central en la nueva gestión biopolítica de la infancia - especialmente la más pobre-, al aportar cierto sello en lo que Castel (2003) llama "la nueva cuestión social". No solo habla de un desplazamiento en términos de sus condiciones de emergencia, sus lugares de inscripción, los sujetos que produce y sus efectos políticos, sino también en sus formas de interpretación, representación y, con ello, en sus formas de afrontarla. Así creemos que los dispositivos de intervención recogen y actualizan un desplazamiento en la matriz de interpretación de la pobreza que va de una de tipo social/económica/política asociada a una "cultura de la pobreza" (Fassin, 1999) a una "clínica de la pobreza", individual y centrada en las capacidades del sujeto. La pobreza se singulariza en políticas sectoriales desvinculadas de las políticas macro y de medidas estructurales que condicionan la efectividad de las primeras (Rodríguez, 2013).

\section{Tecnologías literarias: el Manual para el apoyo y seguimiento del desarrollo psicosocial de los niños y niñas de 0 a 6 años y la construcción de objetos y factualidades}

El Manual para el apoyo y seguimiento del desarrollo psicosocial de los niños y niñas de 0 a 6 años (CCC, 2008) es un instrumento elaborado por el Programa de Apoyo al Desarrollo Biopsicosocial del sistema de Protección a la infancia Chile Crece Contigo, cuyo fin es brindar a los equipos de salud que intervienen directamente con niños, niñas y sus familias orientaciones y recomendaciones que, en palabras del propio manual, "contribuyan a fortalecer su liderazgo técnico para apoyar el desarrollo de los niños y las niñas" (CCC, 2008: 5). Se trata de un material de 102 páginas elaborado a partir de la "experiencia de los equipos de salud de la atención primaria, y a la evidencia científica de la efectividad de las intervenciones" (CCC, 2008: 5) y que no presenta autoría. Así dicho, el manual se muestra como una herramienta que representa hechos objetivos desvinculados de cualquier referencia a sus propias condiciones de emergencia, y como voz de un aparato estatal que trasciende a un sujeto o un proyecto político en particular.

Si pensamos en el trabajo de Shapin y Shaeffer (2005) sobre la vida experimental y su hipótesis de que en la construcción de un hecho científico se ponen en juego una tecnología material, social y literaria, cabe pensar en el manual como una tecnología literaria, es decir, como un elemento constitutivo "en la producción de hechos y en la protección de estos hechos frente a los elementos del conocimiento que se pensaba que podían generar discordia y conflicto" (Shapin y Shaeffer, 2005: 49). Por lo tanto, vemos que el análisis de los manuales que materializan las políticas de salud infantil desde una perspectiva discursiva, junto con permitirnos acceder a los significados por medio de los cuales el Estado y sus dispositivos construyen sus definiciones de infancia - normal o anormal, vulnerable, en riesgo, etcétera-, abre la posibilidad para que estos manuales sean 
pensados como entidades que ejecutan acciones, y es esa capacidad de acción lo que Austin (1982) llama "performatividad". El manual como instrumento performativo sugiere que en él mismo se despliegan reglas, convenciones y estrategias discursivas que construyen los hechos que dice representar. Como afirma Martínez:

Mientras que en la ciencia instituida el lenguaje es concebido como un medio meramente descriptivo, transparente y neutral, que sencillamente "refleja" la realidad (Tuffin, 2005), el desplazamiento hacia una perspectiva discursiva concibe al lenguaje como constitutivo, dinámico y activamente involucrado en la construcción de la realidad. (Martínez, 2010: 33).

Para la teoría de los actos de habla (Searle, 1977), los enunciados sirven no solo para expresar proposiciones, sino también para realizar acciones lingüísticas sujetas a un conjunto de reglas convencionales, cuya infracción afectará directamente a los efectos comunicativos. Así Searle afirma: "Argüiré que cosas tales como plantear preguntas o hacer enunciados están gobernadas por reglas de manera completamente semejantes a aquellas en las que lograr un tiro de béisbol o mover un caballo en el ajedrez son formas de actos gobernados por reglas" (Searle, 1977: 2).

Desde esta perspectiva, los manuales profesionales pueden ser definidos como un conjunto de actos de habla o un macroacto de habla, según Van Dijk (1996), en el que se articulan de modo privilegiado, y siguiendo a Austin (1982), enunciados de tipo performativos, asertivos, directivos y declarativos, y que por medio de instrucciones intentan mostrar "un registro meramente descriptivo de fenómenos dados, preexistentes e independientes del discurso que da cuenta de ellos" (Martínez, 2012: 40). Esto se haría a través del uso de terminologías que, de acuerdo a Cabré:

Los tecnólogos o los profesionales de cualquier ámbito requieren términos para representar y expresar sus conocimientos, para informar, transferir o comprar y vender sus productos. No existe especialidad alguna que, como hemos dicho antes, no posea unidades específicas que denominen sus conceptos. $\mathrm{Y}$ estas unidades son los términos. $\mathrm{Y}$ el conjunto de los términos usados específicamente en un ámbito constituye la terminología de este ámbito (Cabré, 2011: 2).

Si bien, para esta autora, las terminologías están destinadas a satisfacer una necesidad social relacionada con la información y la comunicación, pensamos que cumplen un papel cardinal en la construcción misma de los fenómenos que se quieren estudiar, cuestión que Potter (1998) inscribe dentro de lo que llama la "construcción de factualidades" y Woolgar las "formas en cómo el discurso constituye sus objetos" (Woolgar, 1999: 104). Para este último, el fenómeno no tiene una existencia independiente de sus medios de expresión, de sus recursos de expresión y persuasión.

La producción de un manual depende de varios procesos de escritura, lectura y lo que Latour y Woolgar llaman "inscripción literaria": 
La función de la inscripción literaria es persuadir a los lectores, pero los lectores solo se convencen completamente cuando parecen haber desaparecido todas las fuentes de persuasión. Dicho de otro modo, los participantes consideran que las diversas operaciones de escritura y lectura que sostienen un argumento son en gran medida irrelevantes para los "hechos", que tan solo surgen en virtud de esas mismas operaciones. Hay, por tanto, una congruencia esencial entre el "hecho" y el funcionamiento con éxito de diversos procesos de inscripción gráfica. (Latour y Woolgar, 1985: 91).

Lo anterior permite preguntarse qué estrategias retóricas utilizan los autores invisibles para lograr que sus textos circulen entre la comunidad experta y entre el público en general como verdades indiscutibles.

Por otra parte, se sabe que todo discurso implica una dimensión retórica, y el discurso disciplinario no es la excepción. El lenguaje constituye una condición indispensable para la existencia del propio programa de intervención, así como de las disciplinas que este reúne. No obstante, siguiendo una orientación pragmática del discurso (Potter, 1998), compatible a su vez con una visión foucaultiana que concibe el discurso como práctica social, se debe considerar que el manual solo funciona como tal dentro de una red o dispositivo mayor de gobierno, el cual permite brindar de autoridad social a los actos de habla de un discurso, en este caso al manual, en tanto parte de diversas prácticas tecnocientíficas que permiten invisibilizar o cajanegrizar (Latour,1999) la producción social de las "descripciones" científicas que contiene (Bourdieu, 1985; Potter, 1998).

\section{Material y método}

Se llevó a cabo un estudio cualitativo, exploratorio e interpretativo de diseño circular-emergente (Flick, 2004; Valles, 2000), sensible a cómo los diversos elementos del corpus en análisis pueden modificar los tópicos de investigación previamente definidos. El estudio fue guiado desde el análisis de discurso. Por discurso debe entenderse la comprensión del lenguaje en uso, es decir, con la facultad tanto de promover como de coartar determinados tipos de relaciones sociales (Íñiguez-Rueda, 2006).

De modo particular, la presente investigación fue desarrollada desde la perspectiva del discurso de M. Wetherell y J. Potter (Potter, 1998; Potter y Wetherell, 1987; Wetherell y Potter, 1996). Este enfoque de análisis discursivo, que integra los desarrollos de la filosofía analítica, la etnometodología y el posestructuralismo, entre otros, se caracteriza por orientarse hacia el lenguaje en uso, lo cual implica enfocarse fuertemente en los componentes performativos y pragmáticos del discurso con respecto a la realidad social (Íñiguez-Rueda, 2006; Wetherell y Potter,1996).

Estos autores estipulan tres herramientas analíticas principales para este enfoque de análisis de discurso: la función, la variabilidad de los discursos y los repertorios interpretativos. De acuerdo a Wetherell y Potter, por función debe entenderse el objetivo pragmático del discurso analizado, el cual conjuga, en la nomenclatura de 
John Austin (1982), tanto la dimensión estrictamente ilocucionaria del discurso - sus efectos como acto de habla - como sus efectos perlocusionarios; es decir, aquellos que van más allá de la situación inmediata y que lo ligan al contexto social en que se originan y circulan como prácticas sociales (Wetherell y Potter, 1996). Estas funciones del discurso si bien son el objeto fundamental del análisis, no son inmediatamente pesquisables. Para llegar a su develamiento, Potter y Wetherell señalan que deben observarse las variabilidades del discurso, o sea, las formas diferenciales y muchas veces paradójicas de establecerse los sujetos y objetos del discurso, lo cual es complementado, finalmente, con el establecimiento de los repertorios interpretativos.

Los repertorios interpretativos, noción creada por Gilbert y Mulkay (1984) en sus investigaciones etnográficas de laboratorios para referir la construcción de hechos científicos, se entienden como "los elementos esenciales que los hablantes utilizan para construir discursos, conformándose como restringidas gamas de términos que derivan de una o más metáforas clave (...). La presencia de un repertorio está señalada por ciertos tropos o figuras del discurso" (Potter y Wetherell, 1987: 149).

El corpus seleccionado corresponde al Manual para el apoyo y seguimiento del desarrollo psicosocial infantil del año 2008, definido como un texto de apoyo a la labor profesional en atención de salud en el contexto del sistema de protección de la infancia CCC. En relación a este corpus, dos son los criterios que de acuerdo a Iñiguez y Antaki (1998) permiten identificarlo como un discurso: representatividad y efectos discursivos. Por representatividad, estos autores se refieren al hecho de que los participantes del discurso actúan en su rol como representativos del grupo o comunidad que el/la investigador/a identificó como protagonistas en la relación social, y por tanto no son sus cualidades personales las relevantes, sino el hecho de que son miembros del grupo o comunidad en cuestión. Por el contrario, los efectos discursivos corresponden a aquellos actos lingüísticos que se despliegan en el texto y que se imponen a los sujetos, sin presuponer intencionalidad de un agente particular (Íñiguez-Rueda, 2006; Íñiguez-Rueda y Antaki, 1998).

El análisis de discurso guiado por este método establece que, tras sucesivas lecturas del material, y siguiendo el principio de la variabilidad, se establezcan diversos temas o hipótesis de lectura, los cuales, tras nuevas lecturas, se irán constituyendo como repertorios interpretativos construidos en base a una función determinada, es decir, a qué acción social configura el discurso (Potter y Wetherell, 1987; Wetherell y Potter, 1996). Por tanto, en concreto, el equipo de investigación emprendió sucesivas lecturas del material para codificar temas preliminares que correspondían a extractos del texto escogido, con el propósito de contar con un volumen manejable de corpus de estudio. Posteriormente, este material fue analizado discursivamente, determinando referentes y la variabilidad de temas, a fin de establecer los diversos repertorios interpretativos que vehiculizaran, a su vez, distintas funciones lingüísticas y versiones de mundo (Potter, 1998; Potter y Wetherell, 1987). 


\section{Resultados}

\subsection{Repertorio de los cimientos de lo social: la relación como actor}

El primer repertorio interpretativo identificado consiste en posicionar las relaciones sensoriales entre cuidador e infante como fundamentos del orden social, lo cual se produce lingüísticamente a través de dos operaciones relacionadas: establecer de un determinado modo estas sensaciones e invisibilizar a los sujetos que las ejecutan - es decir al (la) cuidador(a) y al infante-, a través del uso de un lenguaje fáctico (Potter, 1998). Obsérvese este primer fragmento del manual:

El significado social de las experiencias sensoriales y motoras - sonidos, particularmente de la voz humana, la música, el ruido, la luz, los colores y las formas, particularmente los rostros, presión o roce sobre la piel, los olores, particularmente de las personas, los sabores, etc.- constituyen [sic] la base de las relaciones con las otras personas y con el medio ambiente a lo largo de la vida, así como del reconocimiento de las diferencias individuales en el complejo proceso de cambios. (CCC, 2008: 13).

Obsérvese ahora una variación de la misma frase, producida unos párrafos más adelante:

El significado social de estas experiencias constituyen [sic] la base de las relaciones con figuras significativas, con otras personas y con las instituciones a lo largo de la vida, determinando la tendencia de usar o no la violencia, el respeto o no de las diferencias y la equidad o inequidad en las relaciones entre hombres y mujeres. (CC, 2008: 13).

Vemos que este párrafo comienza con un error gramatical que no permite, en un primer momento, determinar si el sujeto de la oración es "el significado social" o las "experiencias sensoriales y motoras"; luego, la siguiente conjugación verbal ("constituyen") nos permite apreciar que el agente de la frase son las experiencias, las cuales son relacionadas con diversos eventos, tal como se puede apreciar también en los siguientes fragmentos:

Los lazos afectivos tempranos constituyen la base para el desarrollo de las capacidades cognitivas, la adquisición del lenguaje y la identificación empática con otros seres humanos. (...) La ausencia de estos cimientos básicos de la vida se asocia a nutrición inadecuada, crecimiento inapropiado, enfermedades frecuentes, rendimiento escolar y laboral deficientes, y acumulación de limitaciones en el potencial para asumir mayores responsabilidades en la comunidad. (CCC, 2008: 14).

Los lazos afectivos son descritos como eventos que, ya sea por acción - como en el primer párrafo citado-, o por ausencia —en el segundo párrafo-, se posicionan como agentes a partir de la figura metafórica de las bases o los cimientos de lo social. Los eventos que, a su vez, son "cimentados" por los lazos afectivos involucran gran parte de la biografía del sujeto infante - pero, como 
dijimos al principio, no es mencionado en ningún momento en los textos, así como tampoco lo es su cuidador(a) - y abarcan unos variopintos sucesos ordenados y jerarquizados desde lo biológico, ligados incluso a dimensiones madurativas (nutrición, crecimiento), a sucesos ligados a la inserción social del individuo (como su "potencial" para asumir mayores responsabilidades en la comunidad). De este modo, a través de estas declaraciones, se disponen, en el manual, dos tipos de continuidades: entre lo biológico-psicológico y social, por un lado, y entre el cuerpo de la madre y el del infante, por otro.

Como se aprecia, estos eventos biológico-sociales cimentados por los lazos afectivos son presentados en los textos bajo un estricto orden causal y unidireccional, desde los individuales a los colectivos. A su vez, puestos los lazos como bases, su ausencia se asocia - vínculo correlacional que implícitamente es puesto como causal - a una serie de "amenazas" que también se desplazan de lo individual a lo social:

El desarrollo psicosocial y la salud mental de los niños o las niñas y adolescentes son fundamentales para el bienestar y calidad de vida de la población y para la calidad de la convivencia social (CCC, 2008: 14).

Por otra parte, también se puede argumentar que:

Las inequidades que se presentan en el desarrollo infantil temprano tienen la capacidad de amenazar las competencias requeridas para vivir en sociedad (CCC, 2008: 8).

En suma, no solo se produce en todos estos párrafos la reducción y ajuste de lo social a lo interpersonal (Burman, 1998), sino que lo que es cimentado y encadenado son adaptaciones sin sujetos, vale decir, son - como se afirma en el último fragmento citado- competencias desprovistas de todo contexto, e incluso de sus propios ejecutantes. Con esto se despliega un tipo de construcción de la infancia que se traduce en capacidades abstractas y "blandas" (usando el lenguaje de las competencias), y en factores también abstractos que producen un riesgo de exclusión. Asimismo fragmentan el espacio del bienestar "en una multiplicidad de comportamientos, zonas y pliegues de riesgo" (Bianchi, 2012: 88).

\subsection{EI Estado como agente de racionalidad, salvación y unidad nacional}

Uno de los avances más significativos del siglo pasado en el ámbito de la salud pública en Chile fue la disminución de la mortalidad infantil. Al inicio de los años setenta, este indicador entra en franco descenso, el que ha sido sostenido durante las últimas décadas, alcanzando en el año 2005 un 7,9 x 1000 nacidos vivos. Para el país, el desafío actual es disminuir la mortalidad infantil a 7,5 × 1000 nacidos vivos al 2010 y a 5,3 x 1000 nacidos vivos al 2015, pero especialmente el desafío es favorecer el óptimo desarrollo integral de los niños y las niñas para mejorar su calidad de vida [...]. Por otro lado y respondiendo a las orientaciones de la Organización Mundial de la Salud, el país ha decidido 
orientar las acciones del sistema de salud hacia un enfoque de los determinantes sociales de la salud. (CCC, 2008: 7).

Otro actor del corpus es el propio Estado. La cita anterior corresponde al primer capítulo del manual, en el que se presentan los "antecedentes conceptuales". Estos antecedentes establecen tanto un preámbulo para el propio texto como para la puesta en marcha del sistema Chile Crece Contigo en el año 2006.

Como se puede apreciar en ambos extractos, el país — aquí considerado como equivalente al Estado o, como ocurre en otras partes del manual, a figuras como "el Gobierno"- emerge como el sujeto protagonista de una historia social de desarrollo y progreso sanitario - obviando a otros actores, como las mismas mujeres-, y que toma y ejerce decisiones "orientado", ya sea por la "evidencia" científica como por las políticas internacionales.

Esta especie de declaración de principios sobre el sentido de la política pública, de la cual la voz del manual actúa en cierto sentido como un "testigo modesto" (Haraway, 2004), no solo presenta al Estado como actor central, sino como un actor racional guiado a su vez por el saber científico. Ahora, desde los estudios sociales de la ciencia, en particular desde su vertiente discursiva (Gilbert y Mulkay, 1984), se ha analizado cómo el discurso científico es tributario de un particular tipo de retórica de construcción de factualidades, que usa estrategias como la comparación, el listado de cotejos o la formulación de casos extremos, entre otras (Edwards y Potter, 1992; Potter, 1998; Potter y Wetherell, 1987). El uso de la factualidad se presenta claramente en la invocación que se hace continuamente en el manual de las "evidencias" para apoyar diversas decisiones del Estado supuestas o explícitas en el manual. Acá se presenta un ejemplo, el cual sigue a la primera cita presentada en este apartado:

A partir de la década de los 80 , los avances científicos, particularmente en el ámbito de las neurociencias, han permitido una mayor comprensión de los procesos de desarrollo cerebral y del sistema nervioso y sensorial, evidenciándose la importancia vital de la vida intrauterina y de los primeros años en la maduración biológica, que permite a los individuos llegar a ser personas integradas en una sociedad. Así mismo, se confirma que esa maduración biológica depende y se produce en interacción con el ambiente humano y físico en el que el niño o la niña crece (CCC, 2008: 7).

Una vez más se produce lingüísticamente una continuidad biológico-social y cierta despolitización de lo social. Es interesante como hipótesis asumir que existe una convergencia sinérgica entre este discurso ilustrado de la ciencia (basada en la figura retórica de la evidencia), que implica un desarrollo desde el no saber al saber, y este discurso que presenta al Estado como actor que moviliza al colectivo social. De esta forma, el discurso del Estado como actor racional entronca simbólicamente con un discurso del Estado como agente benefactor de la población. Es decir, el Estado no solo se nos presenta preformado como un agente racional, sino que cumple una función salvífica encarnado en su accionar en las políticas públicas que adquieren, en la década que se crea el sistema, un fuerte énfasis en el ámbito social. 
Esta imagen racional/benefactora es intercambiable ya sea para el Estado, como figura abstracta del derecho, como para el Gobierno como ejecutor concreto. Véanse los dos siguientes párrafos:

En el año 1990, recuperada la democracia, el Estado de Chile ratificó la "Convención sobre los Derechos del Niño" asumiendo el compromiso de asegurar la protección integral del niño y la niña mediante la interrelación de todas las áreas que inciden en su desarrollo psicosocial. A partir de 1993, se amplía a los niños y las niñas hasta los 5 años la evaluación y estimulación del desarrollo psicomotor. El seguimiento de esta actividad ha permitido demostrar, entre el año 2004 y el 2006, un aumento de la cobertura en la evaluación del desarrollo psicomotor y de las tasas de recuperación de los niños y las niñas con déficit en su desarrollo, de un $44,3 \%$ de recuperación a los 18 meses y un $47,2 \%$ a los 4 años en 2004 , a un $58,2 \%$ a los 18 meses y 58,6\% de recuperación a los 4 años en el año 2006" (CCC, 2008: 7).

El actual Gobierno, asumiendo que "no es aceptable que el lugar en la estratificación socioeconómica que ocupa el hogar en que una persona nació sea uno de los mejores predictores del lugar en esta estratificación que ocupará en la vida adulta”, convocó al consejo asesor presidencial para la Reforma de las Políticas de Infancia. Este consejo entregó, en junio de 2006, el informe "El futuro de los niños es siempre hoy" y en base a este se decidió implementar el Sistema de Protección a la Primera Infancia - Chile Crece Contigo" (CCC, 2008: 8).

En cuanto a las funciones lingüísticas, la presentación del Estado como agente racional se refuerza en su calidad de agente ético, que sitúa su accionar desde un marco benevolente y de justicia. Se muestra incuestionable políticamente, pues estaría más allá de la política, al despojar a las políticas de infancia de cualquier rasgo deliberativo o conflictivo. La infancia es una figura que le permite al discurso estatal situar al Gobierno como protagonista de la cohesión política. El proyecto de sociedad no es discutible en un país altamente desigual y estratificado. Siguiendo a Anderson (1993), la invocación al "futuro de los niños" sigue la genealogía moderna de la nación en tanto "comunidad imaginada," que aglutina a un todo social sin fracturas o antagonismos. Se da en un contexto más amplio en que la figura de la presidenta Bachelet - gestora del proyecto(mujer, madre e hija de un general asesinado) viene a representar en el imaginario político la unidad nacional.

\subsection{La operación del manual: el Estado como coach}

Esta primera voz del Estado, más bien presentada dentro de un contexto, luego es introducida al manual. En los capítulos más "prácticos" del mismo, se produce una curiosa forma lingüística de ventriloquía textual, en la cual el manual habla a un público trasuntando la propia voz del Estado:

2.4.1.2. Recomendaciones al equipo de salud de mensajes a transmitir en los contactos con la madre, el padre o cuidador(a) de un niño o una niña de 0 a 1 Año. Comprenda y responda a las necesidades de su hijo o hija. 
Usted no va a malcriar a su hijo/hija cuando lo consuela porque llora o está inquieto. Responda a las señales de llanto, sueño o risa de su hijo o hija, le puede enseñar a él o ella a tener una sensación de confianza básica en usted y en el mundo.

Ignorar el llanto puede causarle altos niveles de estrés, tanto al niño o la niña como a sus cuidadores(as).

La crianza es un proceso de aprendizaje, tanto para el cuidador(a) como para el niño o la niña.

Si a su hijo o hija le gusta lo que usted está haciendo, quiere decir que usted lo está haciendo bien, tenga confianza. (CCC, 2008: 20).

En primer término, apuntemos que nos encontramos ante un listado, el cual es, junto con la citación de "evidencias" también usado en el manual, un dispositivo de construcción de factualidades (Edwards y Potter, 1992). En este sentido, se debe entender que estas "instrucciones" implican una cadena de relaciones que va desde los profesionales hasta las familias, y ello las convierte en una técnica de poder que opera aún con dos acciones: asume la libertad del sujeto, pero al mismo tiempo la coarta a través del dispositivo de instrucciones - presentado como "recomendaciones"-. De este modo, se opera un dispositivo de poder ya clásicamente usado para adoctrinar a las madres: asumir que el o la cuidadora sabe cuidar al infante, pero aun así operar como si no lo pudiese hacer. Dan "consejos profesionales" y mezclan, de este modo, lo disciplinario con técnicas de gestión.

\subsection{La figura de la madre: del sujeto en riesgo al sujeto de riesgo}

Como ha sido consignado, la figura de la madre aparece aludida en el manual en diversas formas, ya sea interpelada como sujeto de "recomendaciones" profesionales o desde actos declarativos que cumplen una función inclusiva; es decir, que la consideran como sujeto de necesidad para la política pública.

No obstante, desde la racionalidad estatal que se pone en juego en el texto, las madres son también tipificadas en cuanto a si actúan o no como agentes de riesgo para los niños. Como afirma Sepúlveda (2015), uno de los modos a partir del cual el riesgo puede operar en tanto racionalidad y dispositivo tecnológico es catalogando a los sujetos, lo que conlleva un paso subrepticio desde la consideración de un "sujeto en riesgo" hacia una del sujeto "de riesgo" para los demás. Esta lógica se despliega también a propósito de determinadas conductas o experiencias de la madre que podrían afectar a su hijo/a, tal como se ve en los siguientes fragmentos:

Las mujeres que viven violencia de parte de su pareja, o las que pertenecen a hogares uniparentales, con jefatura femenina o aquellas que no comparten la responsabilidad del embarazo y la crianza con el progenitor, están expuestas a mayores niveles de estrés, ansiedad o depresión, que puede afectar la crianza y el desarrollo del niño o la niña (CCC, 2008: 10).

Una madre deprimida presenta una disminuida o sensibilidad necesaria para entender las señales del hijo o hija, de responder ante ellas de forma suficientemente 
buena y rápida, y de tolerar las exigencias del cuidado intenso de un recién nacido. Una madre con dificultades en regular o tolerar la expresión de afectos negativos o el llanto de su bebé puede tener interacciones de carácter negativas que vayan en aumento si no se interviene; así, el deterioro de las interacciones entre madres deprimidas y sus hijos o hijas puede tener consecuencias desfavorables en el desarrollo emocional y cognitivo del niño o niña (CCC, 2008: 41).

Nuevamente, desde un discurso fáctico, el manual ahora opera una construcción del sujeto femenino en una especie de escala de devaluación, compuesta de conductas que las harían más proclives a convertirse ellas mismas en agentes de riesgos u obstaculizadoras para el desarrollo infantil. Esta condición, a su vez, comprende -al igual que en el repertorio interpretativo de la relación como actante - de una graduación del peligro, situación que en este caso es declarada sin la correspondiente presentación de evidencias.

\section{Conclusión y discusiones}

Las producciones discursivas desplegadas por el manual están construidas a partir de un ensamblaje de repertorios que actúan como una matriz de inteligibilidad de la infancia, en la cual la intervención psicosocial - diagnósticos, clasificaciones e intervenciones - legitima y se sitúa como un saber experto. Vemos que estos repertorios expresan una serie de cuestiones sociotécnicas y políticas que operan en el mismo dispositivo de intervención, y es la relación sensorio-afectiva primaria entre niño y cuidador el elemento central en el cual el resto de las dimensiones se van anudando para tejer cierta definición de infancia. La familiarización e individualización que supone situar en la relación afectiva primaria expresa no solo un "olvido de lo comunitario", en palabras de Pacheco y Palma (2014), o cierta vuelta a un modelo asistencial, sino que es un proceso de exclusión de las mediaciones sociocomunitarias que actúan en la producción de problemas sociales, así como en la producción de alternativas de salud y bienestar.

Por otro lado, el manual construye a un Estado como autoridad exclusiva sobre los relatos de la infancia, no solo desde una posición de saber asimétrica respecto a la experiencia de crianza de los actores convocados (madres y padres), sino que obtura los conflictos de interpretación $\mathrm{u}$ oposición por medio de recursos lingüísticos que van desde el lenguaje fáctico —al despersonalizar la presentación de evidencias y listados - hasta el posicionamiento del Estado como actor exclusivo de los logros sanitarios en el país. Esto se vuelve principalmente problemático en un contexto en que urge democratizar las relaciones de saber y las formas de pensar e intervenir en la infancia. La tecnocracia, esta vez vestida con los ropajes de la cientificidad y la salvación, ensombrece tanto la visibilidad de los saberes de las usuarias del programa como de los mismos profesionales que intervienen.

Los fuertes usos de los recursos fácticos despolitizan la misma construcción de los sujetos de intervención, ya que cuestiones como las relaciones de poder de género o generación, las relaciones de clase o, incluso, las de etnia quedan invisibilizadas por medio de retóricas higiénicas y naturalistas como "contexto", 
"ambiente" o "factores estresores". Como destaca Galaz y Guarderas, la intervención construye "ciertos mundos posibles de ser vividos" (Galaz y Guarderas, 2017: 73) por los sujetos de intervención para poder ser incluidos en el accionar de estos dispositivos. Estos mundos son, en el caso de este programa analizado, mundos despolitizados si pensamos, siguiendo a Mouffe (1999), que lo político es tensión, disenso y conflicto; muy cercano a la idea de una nuda vida de acuerdo a Agamben (2002). De este modo, la gestión política de la pobreza infantil $\mathrm{y}$ del sujeto pobre infante supone su nominación como sujeto vulnerable per se y en desarrollo, desde un imaginario biológico y en que se apela enérgicamente a la voluntariedad, interés y estado emocional de las madres, que se revela como un factor de riesgo para la propia infancia.

Asimismo, estas producciones discursivas revelan tensiones entre un esencialismo respecto a los rezagos del desarrollo y su necesaria correlación positiva con los vínculos familiares, y una construcción social situada en las prácticas de crianza, en tanto son estas las que permitirían transformaciones importantes en la condición psicosocial de los niñas y niñas. Además se presentan como producciones en las que se conflictúan comprensiones de tipo científico y conocimientos cotidianos.

Finalmente, cabe destacar que el trabajo aquí presentado fue un acercamiento preliminar a una de las múltiples maneras en las que la infancia se performa en el discurso estatal, cuestión que claramente presenta sus límites, y desde ahí es necesario medir sus alcances. Pese a ello creemos que los datos son relevantes para las nuevas comprensiones de la infancia en tiempo de crisis de los modelos de bienestar universales, del rol distributivo del Estado, y en que las políticas sociales están fuertemente basadas en tecnologías y discursos de eficiencia, el capital humano y la tecnocracia, promovidos por las instituciones financieras internacionales. Así, los análisis de los discursos de la infancia que construyen las tecnologías de intervención estatal permiten desnaturalizar la responsabilidad y sobrecarga asignadas a las familias más pobres en la producción del bienestar, para repensar otras formas de política de infancia con mayor soporte social. En ese sentido, creemos que es necesario ampliar y comparar estos datos a nivel regional, si además el sistema y sus programas han sido implementados en otros países, con el fin de revelar otras dimensiones, conflictos y variaciones que permitirán ir complejizando nuestras lecturas discursivas de la producción de la infancia pobre.

\section{Bibliografía}

Agamben, G. (2002): El Poder Soberano y la Nuda Vida, en Homo Sacer I., Madrid, Nacional.

Anderson, B. (1993): Comunidades imaginadas. Reflexiones sobre el origen y difusión del nacionalismo, México, Editorial FCE.

Apablaza, M. (2016): “Discursos de diferencia en Educación: Análisis de la Política de Diversidad/Inclusión Chilena", Ponencia presentada en 5to Congreso Americano en investigación cualitativa. 
Astete, M. y P. Vaccari (2017): "Políticas públicas y subjetividades: Lógicas en disputa en la implementación de programas sociales en la comuna de Lota, Chile", Psicoperspectivas, 16, (1), pp. 31-41.

Austin, J. (1982): Cómo hacer cosas con palabras: palabras y acciones, Barcelona, Paidós.

Bianchi, E. (2012): "El problema del riesgo. Notas para una reflexión sobre los aportes surgidos de la usina genealógica en torno al concepto de riesgo en salud mental", Espacios nueva serie. Estudios de Biopolítica, 7, pp. 84-97.

Bourdieu, P. (1985): ¿Qué significa hablar?, Madrid, Akal.

Cabré, T (2011): Terminología y normalización lingüística, Extraído de: https://www.ehu.eus/documents/2430735/2877801/cabret.pdf

Castel, R. (2003): La metamorfosis de la cuestión social. Una crónica del asalariado, Buenos Aires, Paidós.

Crisosto, R y Salinas, C. (2017): "Una red de incertidumbre en la planificación de megaproyectos urbanos: el caso de la ciudad satélite de Alerce, Chile”, Eure, 43(128), 229-249.

Chile Crece Contigo (2006): Sistematización Programa de Estimulación Temprana, Documento de Trabajo, Santiago de Chile.

Chile Crece Contigo (2008): Manual para el apoyo y seguimiento del desarrollo psicosocial de los niños y niñas de 0 a 6 años, Documento de trabajo, Santiago de Chile.

Chile Crece Contigo (2012): Orientaciones técnicas para las modalidades de apoyo al desarrollo infantil, guía para los equipos locales, Documento de Trabajo, Santiago de Chile.

Edwards, D. y J. Potter (1992): Discursive Psychology, London, Sage.

Ehrenreich, B. y D. English (2010): Por tu propio bien. 150 años de consejos expertos a mujeres, Madrid, Capitán Swing.

Flick, U. (2004): Introducción a la investigación cualitativa, Madrid, Morata.

Fassin, D. (1999): "La patetización del mundo. Ensayo de antropología política del sufrimiento", en Vivero, M y G. Garay, ed., Cuerpo, diferencias y desigualdades, Bogotá, Universidad Nacional de Colombia, pp. 31-41

Foucault, M. (2007): Nacimiento de la Biopolitica. Curso en el Collège de France: (1978-1979), Buenos Aires, Fondo de Cultura Económica.

Foucault, M. (2008): Nietzsche, la genealogía, la historia, Valencia, Pre-textos.

Foucault, M (2011): La arqueología del saber, Madrid: SXXI.

Foucault, M. (2012): El nacimiento de la biopolítica, México, Fondo de Cultura Económica.

Galaz, C. y P. Guarderas (2007): "La intervención psicosocial y la construcción de las 'mujeres víctimas'. Una aproximación desde las experiencias de Quito (Ecuador) y Santiago (Chile)", Revista de Estudios Sociales, 59, pp. 68-82.

Gilbert, G. y M. Mulkay (1984): Opening Pandora's box. A sociological analysis of scientists' discourse, Cambridge, Cambridge University Press.

Gómez, E, M. Muñoz y M. Santelices (2008): Efectividad de las Intervenciones en Apego con Infancia Vulnerada y en Riesgo Social: Un Desafío Prioritario para Chile, Terapia Psicológica, 26 (2), pp. 241-251.

González, J., S. Ligueño y D. Parra (2015): "Educación y gubernamentalidad en el Chile neoliberal", Archivos de Filosofia, 9-10, pp. 85-118.

Haraway, D. (2004): TestigoModesto@; Segundo_Milenio. Hombrehembra_conoce_Oncoraton. Feminismo y tecnociencia, Barcelona, Editorial UOC. 
Íñiguez-Rueda, L. (2006): "El análisis de discurso en las ciencias sociales: variedades, tradiciones y práctica”, en L. Íñiguez-Rueda, ed., Análisis del discurso. Manual para las ciencias sociales, Barcelona, Ediciones UCO, pp.83-124.

Íñiguez-Rueda, L. y Ch. Antaki (1998): “Análisis del discurso”, Antrophos, 177, pp.59-66.

Laclau, E y Ch. Mouffe (2004): Hegemonía y estrategia socialista. Hacia una radicalización de la democracia, México, Fondo de Cultura Económica.

Latour, B. (1999): Pandora's hope: essays on the reality of science studies, Cambridge, Harvard University Press.

Latour, B. (2008): Reensamblar lo social. Una introducción a la teoría del Actor-red, Buenos Aires, Manantial.

Latour, B y S. Woolgar (1985): La vida en el laboratorio. La construcción de los hechos cientificos, Madrid, Alianza.

Lepe-Carrión, P. (2015): "Intercultural Education in Chile: Colonial Subjectivity and Ethno-Governmental Rationality”, Journal of education, 3 (3), pp. 60-87.

Llobet, V. (2014): "Reflexiones sobre un malentendido: producción de necesidades infantiles en políticas de protección”, Psicologia em Estudo, 19 (3), pp. 369-380.

Mardones, R. (2015): “Apropiación del espacio público y acción colectiva juvenil: estudio de caso en una ciudad de Chile", Revista Interamericana de Psicología, 49(3), pp. 332-341.

Martínez, A y L. Iñiguez (2012): "La fabricación del Trastorno de Identidad Sexual: Estrategias discursivas en la patologización de la transexualidad", Discurso \& Sociedad, 4(1), pp. 30-51.

Mouffe, Ch. (1999): El retorno de lo político. Comunidad, ciudadanía, pluralismo, democracia radical, Buenos Aires, Paidós.

Pacheco, J. y M. Palma (2014): "El discurso profesional en servicios sociales comunitarios: una aproximación desde la identificación de repertorios interpretativos", Alternativas. Cuadernos de Trabajo Social, 21, pp. 9-28.

Potter, J. (1998): La Representación de la realidad. Discurso, retórica y construcción social, Barcelona, Paidós.

Potter, J. y M. Wetherell (1987): Discourse and social psychology. Beyond attitudes and behavior, London, Sage.

Rivera, G (2016): “Gobernar a la juventud vulnerable: un análisis discursivo de las políticas de empleo juvenil en Chile”, Revista pueblos y fronteras digital, 11 (21), pp. 192-213.

Rivera, G. (2016a): “Gubernamentalidad y políticas de empleo: la construcción discursiva del joven trabajador en Chile”, Última década, 45, pp. 34-54.

Rodríguez, A. (2013): "La infancia latinoamericana y caribeña en medio de la crisis neoliberal", Revista latinoamericana de ciencias sociales, niñez y juventud, 11 (2), pp. 891-897.

Rose, N. (1990): Governing the soul. The shaping of the private self, London and New York, Routledge.

Rose, N. (2007): Politicas de la Vida. Biomedicina, poder y subjetividad en el siglo XXI, Buenos Aires, Unipe.

Rose, N. y J. Abi-Rached (2013): Neuro: The New Brain Sciences and the Management of the Mind, Nueva Jersey, Princeton University Press.

Searle, J. (1977): “¿Qué es un acto de habla?”, Teorema, 15, pp. 46-58. 
Sepúlveda, M. (2015): “Gubernamentalidad y riesgo en el campo de las drogas: análisis de las prácticas de gobierno en torno al problema-drogas en Chile posdictatorial”, Universitas Psychologica, 14 (5), pp. 1707-1721.

Shapin, S. y S. Shaffer (2005): El Leviatán y la bomba de vacio. Hobbes, Boyle y la vida experimental, Buenos Aires, Universidad Nacional de Quilmes.

Silva, P. (2006): "Los tecnócratas y la política en Chile: pasado y presente", Revista de ciencia política, 26 (2), pp. 175-190.

Soto, R. (2016): "Sedentarismo, deporte y la presión biopolítica de vivir saludable: análisis del discurso del sistema elige vivir sano en Chile", Movimento, 22, pp. 391-402.

Valles, M. (1999): Técnicas Cualitativas de investigación social, Madrid, Síntesis.

Van Dijk, T. (1999): Estructuras y funciones del discurso, México, Siglo XXI.

Wetherell, M. y J. Potter (1996): "El Análisis de discurso y la identificación de los repertorios interpretativos", en Gordo, A. y J. López, coords., Psicologías, discursos, poder, Madrid, Visor, pp. 63-78.

Woolgar, S. (1991): Ciencia: abriendo la caja negra, Barcelona, Anthropos. 